\title{
CORRELATION BETWEEN THE ONSET OF BEADLESS CHARACTER OF NANOFIBROUS WEBS AND RHEOLOGICAL CHARACTERISTICS OF ELECTROSPUN POLYMER SOLUTIONS
}

\author{
Jana ZELENKOVA, Petra PEER, Petr FILIP \\ Institute of Hydrodynamics, Czech Academy Sciences, Prague, Czech Republic, EU, \\ zelenkova@ih.cas.cz,peer@ih.cas.cz, filip@ih.cas.cz
}

https://doi.org/10.37904/nanocon.2021.4375

\begin{abstract}
An overwhelming majority of applications of nanofibrous webs requires sufficiently smooth character of nanofibres surface. This character is apart other parameters strongly influenced by a concentration of chosen polymeric material in the solvents. Qualitative attributes roughly depend on the prepared concentration, specifically whether it belongs to one of the four basic regions: dilute, semidilute unentangled, semidilute entangled and concentrated. A concentration separating the latter two regions is often taken as a zero-th approximation indicating an onset of beadless nanofibrous webs. The present contribution uses as a more precise indicator behaviour of so-called phase angle relating viscous and elastic moduli, in other words mutual participation of viscous and elastic components. To this aim three frequently used polymers were used: copolymer of poly(vinylidene fluoride) and hexafluoropropylene (PVDF-co-HFP), poly(ethylene oxide) (PEO) and poly(vinyl butyral) (PVB). For materials exhibiting first a constant behaviour or moderate decrease in a phase angle for lower concentrations, an approximation of the starting concentration is given by a concentration value where a phase angle curve starts to decrease, apparently reflecting a more progressive viscoelastic nature. As shown, such approximation provides relatively very good approximation enabling to eliminate a traditional trial-and-error method.
\end{abstract}

Keywords: Nanofibres, beadless morphology, PVDF-co-HFP, polymer concentration, rheology

\section{INTRODUCTION}

In past decades the application of nanofibrous materials has shifted from classical filters to other uses such as protective clothing, antibacterial wound dressing, antibacterial filtration membranes, and many others. The electrospinning process [1,2] represents one way to produce nanofibres in a relatively cheap manner. This process is based on applying a high voltage (in orders of tens $\mathrm{kV}$ ) to polymer solutions or melts when ejected nanofibres are deposited on a grounded collector. Quality of the resulting nanofibres is also closely related with an appearance of so-called beads along the individual nanofibres which is mostly an unwanted phenomenon. Bead formation strongly depends on various factors such as voltage, tip-to-collector distance, additives as well as a choice of solvents.

One of the crucial parameters is represented by the polymer concentration. Its value contributes to distinguish the visual properties of the final product to three distinct cases: electrospraying (discrete drops), continuous nanofibres and the creation of spots produced by spinning due to incomplete solvent evaporation. De Gennes [3] set four consecutive regions of polymer solutions differing in concentration: dilute, semidilute unentangled, semidilute entangled and concentrated. The first two regions are separated by the so called overlap concentration $c^{*}$, the semidilute regions by the so called entanglement concentration $c_{e}$. For individual materials the multiplicative coefficients $\mathrm{k}$ of entanglement concentration $c_{\mathrm{e}}$ (where a semidilute entanglement region starts) are determined in such a way that for concentrations lower than k.Ce the electrospun nanofibers exhibit singularities (appearance of beads along the nanofibres) and for concentration higher than k. $c_{e}$ the 
beads are suppressed and the nanofibres exhibit a relatively smooth surface. The question is whether this method of evaluating an onset of concentrations for which beadless nanofibres are produced cannot be simplified. To this aim we chose three frequently used materials introduced in the Abstract. These materials are biocompatible and their application as e.g. antibacterial filtration membranes seems to be promising [4].

Based on oscillatory rheological measurements the goal of this contribution is to show a close correlation between the onset of a starting concentration for which a presence of beadless nanofibres dominates and a location of phase angle decrease, i.e. a sudden decrease from the constant course or only a moderate decrease in the curve phase angle vs. the concentration of polymer solution.

\section{MATERIALS AND METHODS}

Kynar Flex ${ }^{\circledR} 2801$ (copolymer poly(vinylidene fluoride)-co-hexafluoropropylene) was purchased from Arkema (France), poly(vinyl butyral) Mowital B 75H was purchased from Kuraray Specialities Europe and poly(ethylene oxide) was purchased from Sigma Aldrich (USA). The solvents N,N'- dimethylformamide (DMF) (p.a.) and ethanol (quality of p.a.) were purchased from P-LAB, a.s. (Czech Republic) and Penta (Czech Republic) respectively. All chemicals were used as obtained without further refinement.

Polymer materials were dissolved in corresponding solvents (Table 1) using a magnetic stirrer MR Hei-Tec (Heidolph, Germany) with the help of a teflon-coated magnetic cross under these conditions: mixing rate was set to $250 \mathrm{rpm}$, temperature $25^{\circ} \mathrm{C}$ and time of mixing was 48 hours (24 hours in the case of Kynar Flex ${ }^{\circledR} 2801$ ). The sufficiently wide and dense concentration range ensured that the full palette of nanofibres was at disposal and moreover, it ensured an acceptable approximation of a location where a phase angle started to decrease.

Table 1 Ranges of concentrations of the individual polymer solution

\begin{tabular}{|c|c|c|c|}
\hline Polymer & Solvent & $\begin{array}{c}\text { Molecular weight } \\
{[\mathrm{g} / \mathrm{mol}]}\end{array}$ & $\begin{array}{c}\text { Concentration } \\
{[\mathrm{wt} . \%]}\end{array}$ \\
\hline PVDF-co-HFP & DMF & $455,000^{\mathrm{a}}$ & $\begin{array}{c}8,10,12,13,15,17,18, \\
20-29 \text { in steps of } 1 \%\end{array}$ \\
\hline PVB & ethanol & $75,000^{\mathrm{a}}$ & $3-10$ in steps of $1 \%, 12,14$ \\
\hline PEO & distilled water & $637,500^{\mathrm{b}}$ & $\begin{array}{c}0.3,0.5,0.6,0.8,1,1.2,1,5,1.8, \\
2-6 \text { in steps of } 0.5 \%, 7,7.5,8,9\end{array}$ \\
\hline
\end{tabular}

${ }^{a}$ molecular weight provided by the producer, ${ }^{b}$ molecular weight determined by SEC (Size Exclusion Chromatography)

The nanofibres were spun (see Table 2 presenting the conditions) using our laboratory needleless device equipped with a high-voltage power supply SL70PN150 (Spellman, USA), a carbon steel stick (10 mm in diameter) with a semispherical hole for depositing of $0.2 \mathrm{~mL}$ of polymer solution and a motionless flat metal collector.

Table 2 Summary of the entry parameters of the electrospinning process

\begin{tabular}{|c|c|c|c|c|}
\hline Material & $\begin{array}{c}\text { Voltage } \\
{[\mathrm{kV}]}\end{array}$ & $\begin{array}{c}\text { Tip-to-collector distance } \\
{[\mathrm{cm}]}\end{array}$ & $\begin{array}{c}\text { Temperature } \\
{\left[{ }^{\circ} \mathrm{C}\right]}\end{array}$ & $\begin{array}{c}\text { Humidity } \\
{[\%]}\end{array}$ \\
\hline PVDF-co-HFP / DMF & 18 & 10 & $23 \pm 1$ & $34 \pm 1$ \\
\hline PVB / ethanol & 20 & 10 & $21 \pm 1$ & $40 \pm 1$ \\
\hline PEO / distilled water & $12-25$ & 20 & $22 \pm 1$ & $39 \pm 2$ \\
\hline
\end{tabular}

A rotational rheometer Physica MCR 501 (Anton Paar, Austria) equipped with the concentric cylinder geometry (the inner and outer diameters were 26.6 and $28.9 \mathrm{~mm}$, respectively) was used both for oscillatory 
measurements (frequency sweep within $0.1-100 \mathrm{~Hz}$ at strain $1 \%$ ) providing elastic $G$ ' and viscous $G$ " moduli, and for shear viscosity measurements (a range $0.01-300 \mathrm{~s}^{-1}$ ). The value of shear rate $\dot{\gamma}=0.12 \mathrm{~s}^{-1}$ belonging to a linear viscoelastic region was chosen for measurement of shear viscosity of polymer solutions with different polymer concentrations, and consequently applied to a determination of specific viscosity. The phase angle $\delta$ ( $\tan \delta=G^{\prime \prime} / G^{\prime}$ ) was determined at frequency of $10 \mathrm{~Hz}$. Temperature was set to $25^{\circ} \mathrm{C}$. Each measurement was carried out at least three times and the individual runs were more or less identical.

A high resolution scanning electron microscope Vega 3 (Tescan, Czech Republic) was used for characterization of nanofibrous mats. Prior to imaging, the samples were sputtered by a conductive coating layer using a sputter Quorum Q150R (Quorum Technologies Ltd, UK).

\section{RESULTS}

Based on measurement of shear viscosities for individual concentrations of PVDF-co-HFP, the specific viscosities were calculated using the relation $\eta_{\mathrm{sp}}=\left(\eta_{0}-\eta_{\mathrm{s}}\right) / \eta_{\mathrm{s}}$, where $\eta_{0}$ is the zero shear rate viscosity (measured at $\dot{\gamma}=0.12 \mathrm{~s}^{-1}$ ) and $\eta_{\mathrm{s}}$ is the solvent (DMF) viscosity ( $=0.807 \mathrm{mPa}$.s). Consequently, the first three concentration regions according to de Gennes [3] were determined separated by the overlap concentration $c^{*}$ $(\approx 12 \mathrm{wt} . \%)$ and entanglement concentration $c_{\mathrm{e}}(\approx 19 \mathrm{wt} . \%)$. These two concentrations correspond to the intersection points of linear segments optimized with respect to the experimental points, see Figure 1.

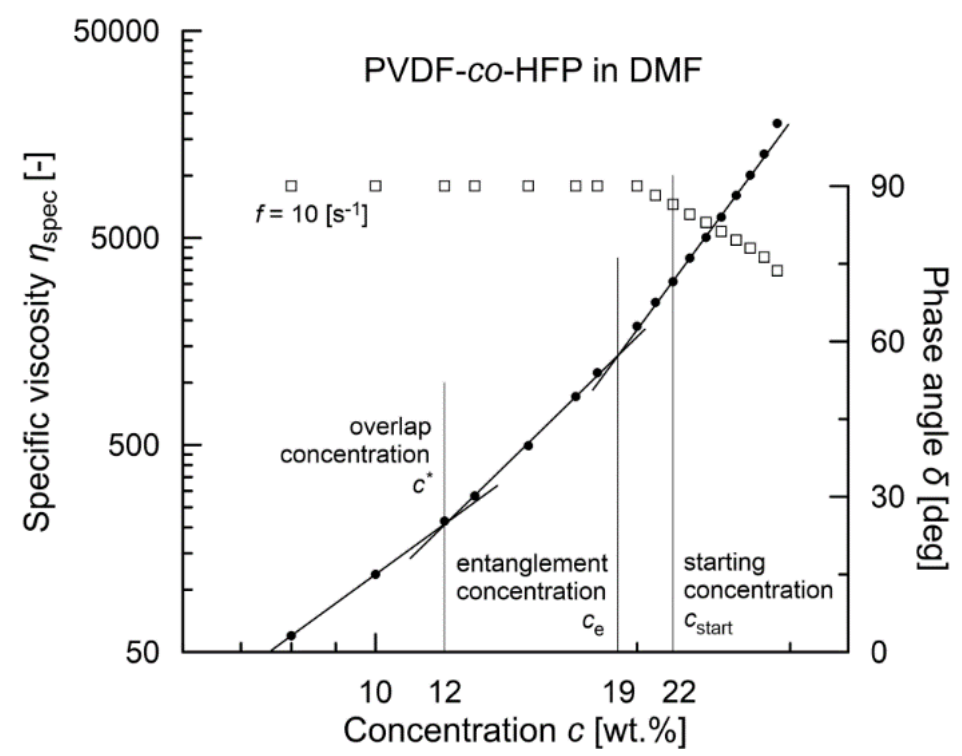

Figure 1 Correlation between an onset of viscoelasticity (a decrease of phase angle, open circles) and a starting concentration corresponding to an onset of beadless PVDF-co-HFP nanofibres

Based on elastic (storage) $G^{\prime}$ and viscous (loss) G" moduli, a course of the phase angle $\delta$ ( $\tan \delta=G^{\prime \prime} / G^{\prime}$ ) is depicted in Figure 1. A value of concentration for which the phase angle starts to decrease (slightly more than 20 wt.\%) corresponds to the formation of network structures among the polymer chains and exceeds a location of the entanglement concentration $(\approx 19 \mathrm{wt} . \%)$.

The morphology of PVDF-co-HFP nanofibres was correlated with the rheological characteristics of measured solutions. Figure 2 documents that up to the overlap concentration $\left(c^{*} \approx 12 \mathrm{wt} . \%\right)$ no nanofibres are formed and as a result only a disordered set of blobs are received. Starting with the overlap concentration the primary forms of nanofibres are indicated mixed with the blobs, and finally a passage from bead to bead-free nanofibres is apparent round the entanglement concentration. It seems that an adequate quality of nanofibres is achieved for $c_{\text {start }}=22 \mathrm{wt} . \%$ and higher. This value closely corresponds to a concentration for which the phase angle starts to decrease as elasticity begins to manifest. 

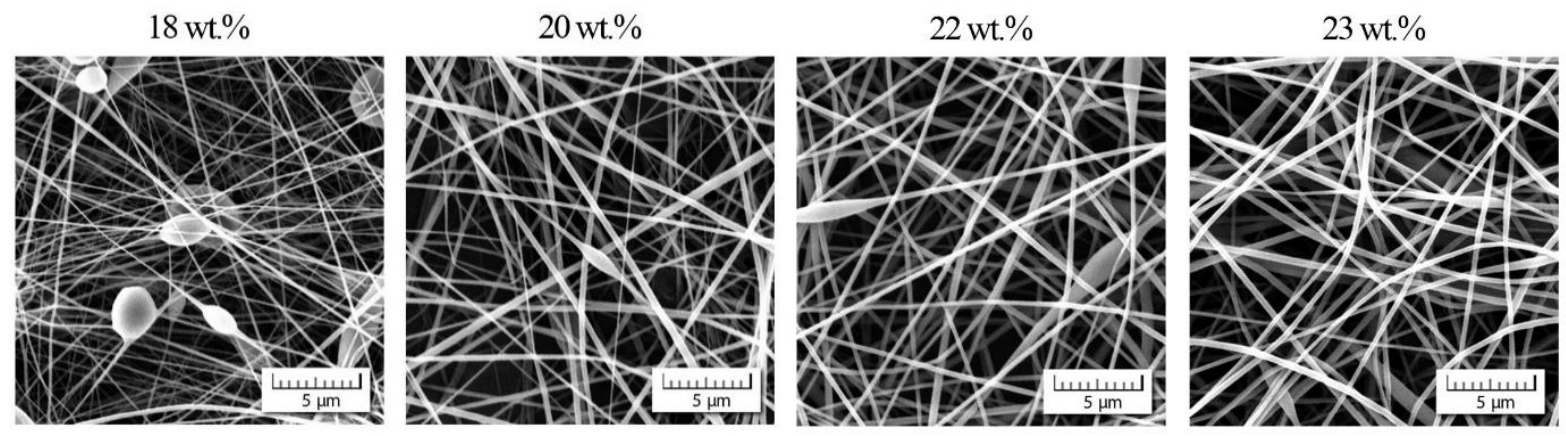

Figure 2 Morphology of individual nanofibres in dependence on PVDF-co-HFP concentration

The analogous experimental approach was carried out with PVB dissolved in ethanol (a measured value $\eta_{\mathrm{s}}=$ $1.087 \mathrm{mPa} . \mathrm{s})$. The results are introduced in Figures $\mathbf{3}$ and $\mathbf{4}$. In this case the starting concentration is slightly lower than the entanglement concentration.

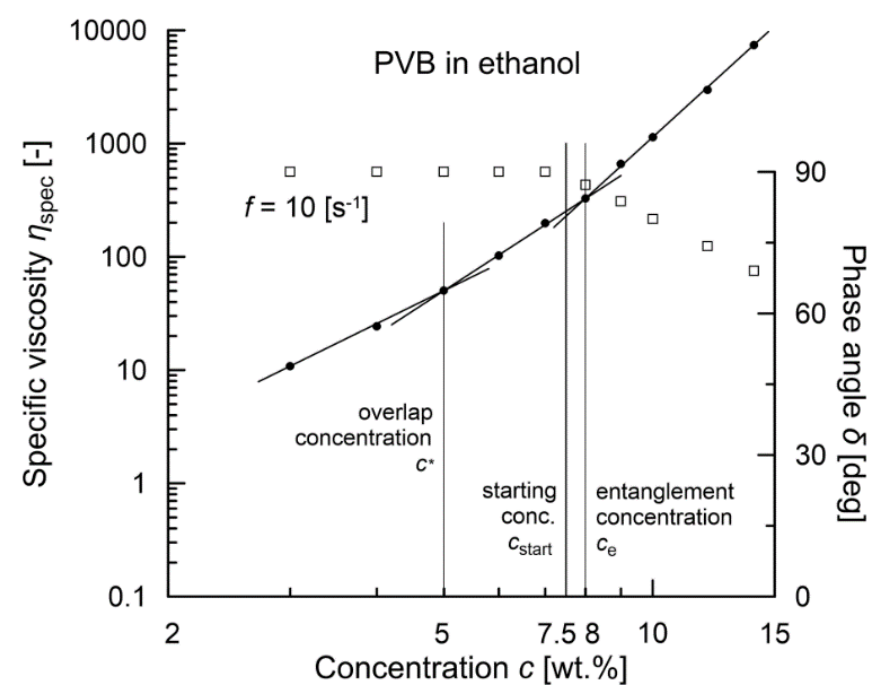

Figure 3 Correlation between an onset of viscoelasticity (a decrease of phase angle, open circles) and a starting concentration corresponding to an onset of beadless PVB nanofibres

5 wt. \%

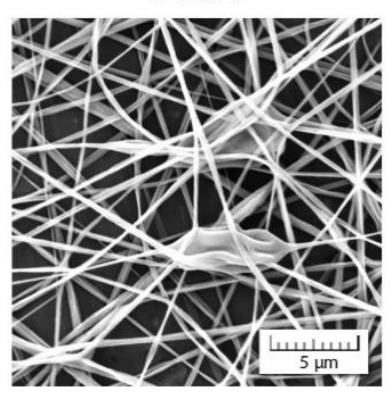

7 wt. \%

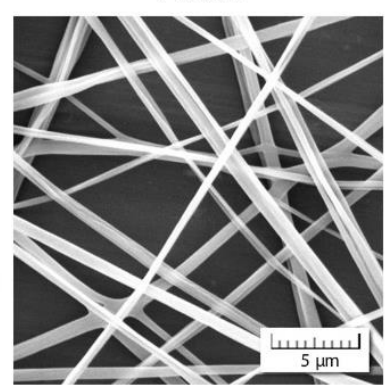

8 wt.\%

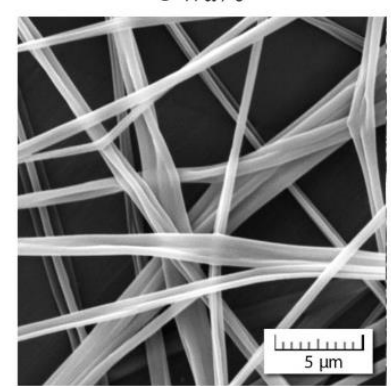

$10 \mathrm{wt} \%$

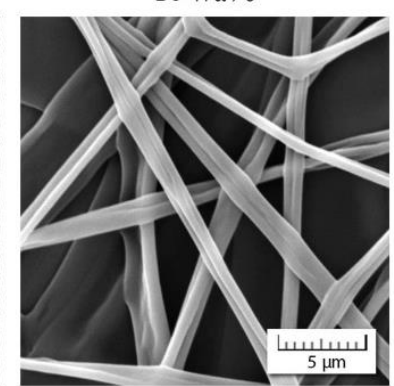

Figure 4 Morphology of individual nanofibres in dependence on PVB concentration

The analogous experimental approach was also carried out with PEO dissolved in distilled water $\left(\eta_{s}=0.890\right.$ mPa.s). The results are summarized in Figures 5 and 6 . In this case $\left(M_{w}=637,500 \mathrm{~g} / \mathrm{mol}\right)$ the polymer solutions exhibit viscoelastic behaviour also for lower concentrations due to higher molecular weight. First, a decrease in the relation phase angle vs. concentration is rather moderate. Then, at the concentration of 
approximately 6 wt.\% a sudden drop is apparent. Hence, this value ( $\left.c_{\text {start }}\right)$ indicates the region from which the nanofibres have dominantly bead-free character.

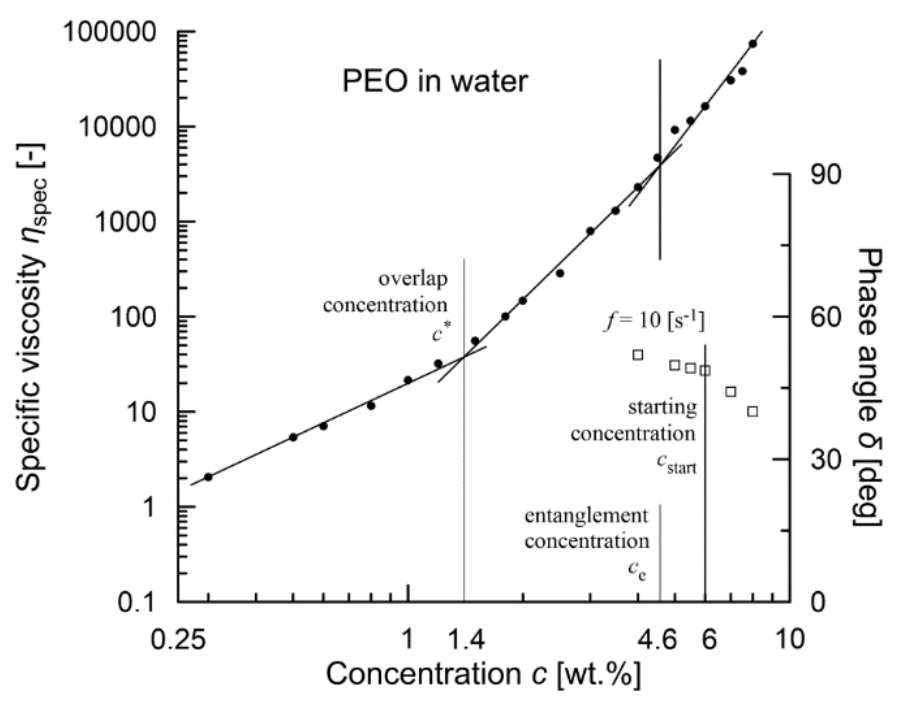

Figure 5 Correlation between a sudden decrease of phase angle (open circles) and a starting concentration corresponding to an onset of beadless PEO nanofibers
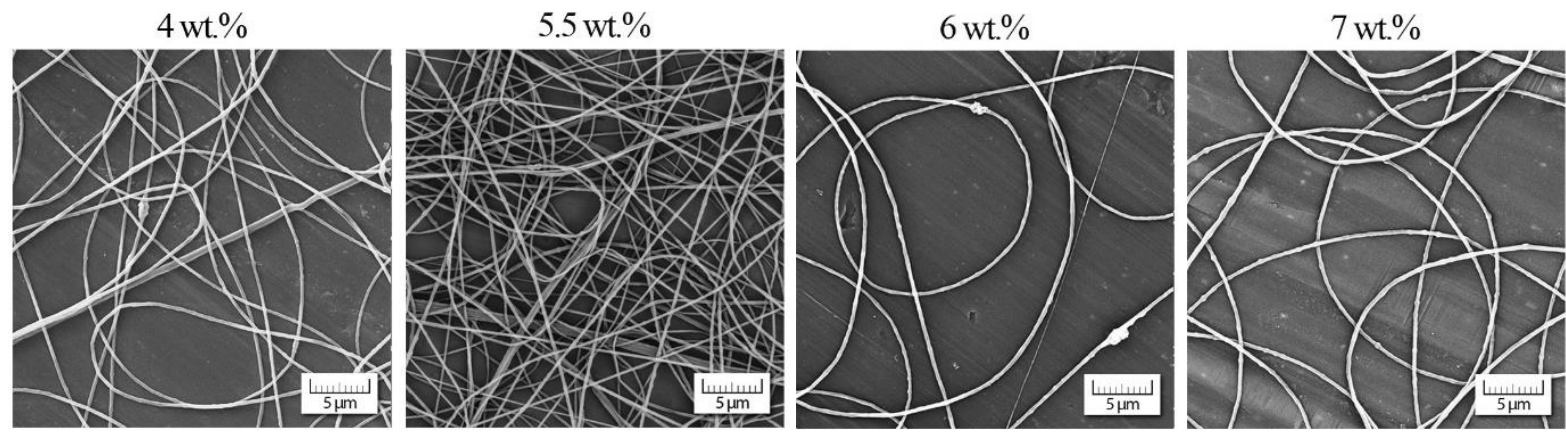

Figure 6 Morphology of individual nanofibres in dependence on PEO concentration

The following Table 3 summarizes the results for all three materials. The differences between the individual polymers also subject to topology of macromolecular chains and their possible entanglement.

Table 3 Summary of the overlap, entanglement and starting concentrations for used polymeric materials

\begin{tabular}{|c|c|c|c|}
\hline Material & $\begin{array}{c}\text { Overlap concentration } \\
\boldsymbol{c}^{\star}[\mathrm{wt} . \%]\end{array}$ & $\begin{array}{c}\text { Entanglement } \\
\text { concentration } \boldsymbol{c}_{\mathrm{e}}[\mathrm{wt} . \%]\end{array}$ & $\begin{array}{c}\text { Starting concentration } \\
\boldsymbol{c}_{\text {start }}[\mathrm{wt} . \%]\end{array}$ \\
\hline PVDF-co-HFP / DMF & 12 & 19 & 22 \\
\hline PVB / ethanol & 5 & 8 & 7.5 \\
\hline PEO / distilled water & 1.4 & 4.6 & 6 \\
\hline
\end{tabular}

\section{DISCUSSION}

The determination of the starting concentration for the PEO solution is not so strict as for the remaining two materials as PEO solutions exhibit viscoelastic behaviour also for lower concentrations due to relatively high molecular weight. A more pronounced decrease of the phase angle starts from already mildly decreasing curve phase angle vs. concentration which contrasts to the PVDF-co-HFP and PVB solutions for which the phase 
angle attains a constant value $\left(90^{\circ}\right)$ for lower concentrations. This also results in relatively gradual passage of PEO nanofibres from beaded to beadless character.

Possible electrospinnability of polymeric materials and quality of the resulting nanofibres is subject to a number of entry parameters which can be roughly distributed into four categories: polymer characteristics (molecular weight, viscosity, etc.), solvent characteristics (rheological parameters, etc.), polymer solution characteristics (concentration, Hansen solubility parameters, etc.), and process characteristics (e.g. voltage, tip-to collector distance, temperature, humidity).

The topic of this study is a participation of polymer concentration on the appearance of singularities (beads) along the electrospun nanofibres. It is well known that characterization of polymeric materials differs from batch to batch [5]. It results in a variable value of the concentration separating beaded and beadless nanofibres. The primary factor causing these discrepancies is represented by variable molecular weight modifying rheological characteristics. It means that the experimental findings valid for one batch cannot be automatically applied for the other and the whole evaluation process should be repeated. The rheological procedure presented in the preceding section substantially accelerates acquiring new data (starting concentrations) with new batches eliminating hitherto necessity to repeat the individual experiments using a spinning device.

\section{CONCLUSION}

Determination of a starting concentration of electrospinnable polymer solutions for which the obtained electrospun nanofibres exhibit a bead-free surface is usually carried out by a trial-and-error method. The proposed method using oscillatory rheological measurements provides a very good approximation of the starting concentrations. For materials exhibiting first a constant behaviour or moderate decrease of a phase angle for lower concentrations, an approximation of the starting concentration is given by a concentration value where a phase angle curve starts to decrease apparently reflecting more progressive viscoelastic nature. It was documented using solutions of three often used polymers: PVDF-co-HFP, DMF and PEO.

\section{ACKNOWLEDGEMENTS}

\section{This research was supported by the Ministry of Education, Youth and Sports of the Czech Republic} (Project LTC 19034). This work was carried out in the frame of the COST Actions CA17107.

\section{REFERENCES}

[1] RENEKER, D.H., YARIN, A.L. Electrospinning jets and polymer nanofibers. Polymer. 2008, vol. 49, pp. 23872425.

[2] XUE, J., WU, T., DAI, Y., XIA, Y. Electrospinning and electrospun nanofibers: Methods, materials, and applications. Chem. Rev. 2019, vol. 119, pp. 5298-5415.

[3] de GENNES, P.G. Scaling Concepts in Polymer Physics. Ithaca, NY: Cornell University Press, 1979.

[4] PEER P., JANALIKOVA, M., SEDLARIKOVA, J., PLEVA, P., FILIP P., ZELENKOVA J., OPALKOVA-SISKOVA, A. Antibacterial filtration membranes based on PVDF-co-HFP nanofibers with the addition of medium-chain 1monoacylglycerols. ACS Appl. Mater. Interfaces (accepted). 2021, vol. 13, no. 34, pp. 41021-41033.

[5] ZATLOUKAL, M.; KOPYTKO, W.; VLCEK, J. The effect of different batches of the same polymer on the flow in flat coextrusion dies. In: Proc. Annual Technical Conf. Soc. Plast. Engnrs. Nashville, TN, USA, 4-8 May 2003; pp. 3509-3513. 